\title{
Finite Element Modal Analysis Of FSC Racing Frame
}

\author{
YU Baojun ${ }^{1}$, SUN Lunjie ${ }^{2}$, BIAN Yahui $^{2}$, SI Sumei ${ }^{2}, Y U$ Wenhan $^{2}$ \\ 1.Changchun University of Technology, Changchun130012,China \\ 2.Mechanical and electronic laboratory, Changchun University of Technology, Changchun130012, China
}

Keywords: FSC frame; Modal analysis; Natural frequency; Vibration mode

\begin{abstract}
The natural frequencies and modes of a structure can be determined by the modal analysis. In this paper, the CATIA software based on the FSC car frame to carry out a three-dimensional modeling, save as STP format. In the Workbench ANSYS, the modal of the frame is solved by the finite element method, and the first 10 order non zero modes and the natural frequency of the frame are obtained. The natural frequency is calculated when the engine idle speed is $33.3 \mathrm{~Hz}$, the normal speed frequency range of $5000-7000 \mathrm{r} / \mathrm{min}$ is $166.7-233.3 \mathrm{~Hz}$, the natural frequency of the finite element method to calculate the first 10 order vibration mode under $100 \mathrm{~Hz}$, so the conclusion of this paper is the frame model of the scheme effectively avoids the excitation frequency of the engine and to avoid the risk of resonance.
\end{abstract}

\section{Introduction}

Formula student China (Referred to as "FSC") is mainly aimed at the undergraduate students and graduate students in various colleges and universities. The event requires teams to design a Formula One car at the time and with limited funding. In any car race driver's safety is the most important, so the frame must be in a variety of extreme conditions can remain safe and reliable. If the car in the course of the car during the resonance phenomenon occurs, it will be on the car's handling stability and reliability of great harm, it is necessary for the frame modal analysis to calculate the natural frequency, that the frame in the car when driving Resonance does not occur.

\section{The principle of modal analysis}

Modal analysis is a kind of modern method that is used to study structural dynamic characteristics.It is the application of system identification method in the field of engineering vibration.Each mode has a specific natural frequency,damping ratio and modal vibration mode. These modal parameters can be obtained by the calculation or test analysis, and such a process called modal analysis calculation or test analysis. In order to obtain modal parameters, it is the characteristic equation of structure vibration characteristic equation of eigenvalue and eigenvector that was obtained by calculation.Both respectively with corresponding natural frequency and the main formation of the structure, as the inherent nature of vibration system, can be obtained from calculation equation to:

$$
\mathrm{M} \ddot{x}+\mathrm{Kx}=0
$$

This formula is an equation of free vibration of $\mathrm{N}$ degree of freedom vibration system.

In the formula:

$$
\begin{aligned}
& \text { M-- mass matrix } \\
& \ddot{x} \text {-- acceleration matrix } \\
& \text { K-- stiffness matrix } \\
& \text { x-- vibration displacement matrix }
\end{aligned}
$$

The solution is in the form of:

$$
\mathrm{x}=\mathrm{A} e^{A} \quad\left(\mathrm{~h}=\mathrm{i} \omega_{0} t \text { 且 } \mathrm{t}^{2}=-1\right)
$$

The $\mathrm{x}$ derivative, 


$$
\begin{aligned}
& \dot{x}=i \omega_{0} A e^{A} \\
& \ddot{x}=-\omega_{0}^{2} A e^{A}
\end{aligned}
$$

Substituting Equation (3) into Equation (1), we can derive the following equation:

$$
\left(\mathrm{K}-\omega_{0}^{2} M\right) \mathrm{A}=0
$$

In the formula:

$$
\omega_{0} \text {--natural frequency }
$$

A--amplitude matrix

If the formula (4) is in accordance with the coefficient determinant:

$$
\Delta\left(\mathrm{K}-\omega_{0}^{2} M\right)=0
$$

Then A is non - zero solution.

According to formula (5) $\omega_{0}$ can be obtained, then $\omega_{0}$ into equation (3), A can be obtained. Therefore, the $\mathrm{N}$ natural frequencies of the $\mathrm{N}$-degree-of-freedom vibration system are $\omega_{01} 、 \omega_{02}, \ldots \omega_{0 n}$, , and the corresponding main formation are $A^{(1)} 、 A^{(2)} 、 \ldots, A^{(n)}$

\section{Establishment of Three-dimensional Model}

Figure 1, it is the use of CATIA software to create the frame three-dimensional model. The material is structural steel. The performance parameters of the material are: elastic modulus $2.05 \mathrm{E} 11 \mathrm{~Pa}$, Poisson ratio 0.29 , yield strength $250 \mathrm{MPa}$, ultimate strength $480 \mathrm{MPa}$.

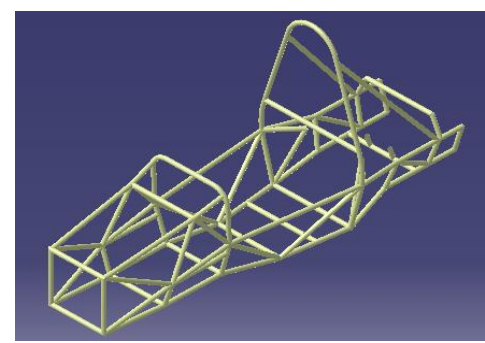

Fig.1 frame 3D model

\section{Import the Frame Finite Element Model}

In this paper, the $3 \mathrm{D}$ frame model of the frame is set up in CATIA software, then the model in CATIA is transferred to STP format and then directly imported into ANSYS. Finally, in ANSYS analysis only need to add the cross-section of each tube, you can divide the grid and then analyze. It should be noted that the imported wireframe model in ANSYS must be a completely broken line segment diagram (the connection between any two points in a three-dimensional wireframe must be a line segment, not a curve that does not break). Otherwise, no result is output after the solution.

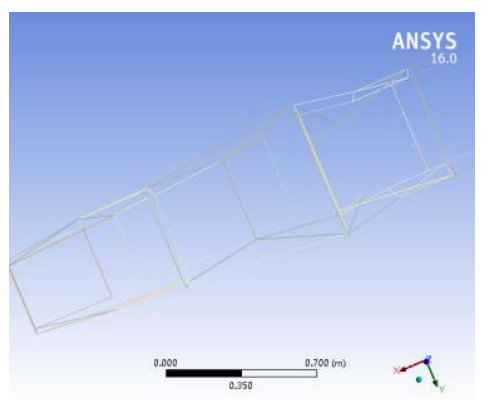

Fig.2 frame 3D model

\section{Division Grid}

Meshing is an important step in ANSYS pre-processing. ANSYS divides the frame into small units by meshing. The density of meshes and the way of meshing affect the quality of meshing and can influence the speed of calculation and the accuracy of calculation. The frame is a truss structure welded by steel pipe. Considering the analysis precision and resource occupancy, Beam 188 is used as the unit analysis type of the frame. In the Workbench, using the Automatic Method in Mesh, and the basic size of the unit to control, the basic size of $0.01 \mathrm{~m}$ beam element grid. Divided into 5078 nodes and 3011 units. 


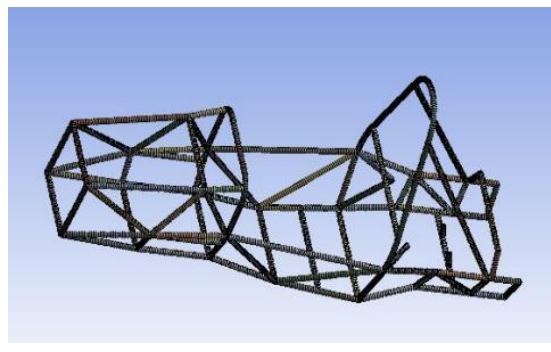

Fig.3 frame mesh

\section{Establish Boundary Conditions}

Pre-Stress set to empty, mainly for Analysis Settings, Max modes to find is used to set the order to be analyzed, this article is set to 10 . The corresponding boundary conditions are set. The boundary conditions have great influence on the results, and the different boundary conditions have different frequencies and modes.

\section{Modal Analysis}

In designing the FSC frame, the designer will not only consider the car bending, torsion, braking, turning four conditions, but also consider the design of the natural frequency of the frame and the engine excitation frequency. This is because when the car on the track to do speed change and experience different road conditions, the driving state is often not only the above four conditions, but the combination of the above conditions. Once the road and the engine excitation frequency and a natural frequency of the same frame, the frame may be in a resonant state, which makes the racing frame of a weak link or the entire frame was destroyed. Therefore, in order to prevent the resonance of the frame, it is necessary to analyze the natural frequencies and vibration modes of the frame, and design a reasonable frame structure according to the analysis results, so that a certain vibration frequency of the car frame itself and road and engine excitation frequency Stagger, so as to avoid resonance. In this paper, Modal function of Ansys Workbench Mechanical module is applied to modal analysis of the frame to obtain the vibration characteristics of the frame at different frequencies, which provides the basis for the frame structure improvement.

In order to analyze the vibration mode of the frame better, the $1-10$ th order non-zero mode of the frame is analyzed in this paper, and its vibration characteristics and frequency characteristics are observed. Table 1 shows the modal analysis frequency.

Table 1 natural frequencies of each mode

\begin{tabular}{cc}
\hline Mode & Frequency/Hz \\
\hline 1 & 0 \\
2 & 0 \\
3 & 0 \\
4 & $4.2953 \mathrm{e}-004$ \\
5 & $4.6392 \mathrm{e}-004$ \\
6 & $1.3802 \mathrm{e}-003$ \\
7 & 33.614 \\
8 & 68.202 \\
9 & 80.283 \\
10 & 93.255 \\
\hline
\end{tabular}

From Table 1, we can see that the natural frequency of the 1-3 order mode of the frame is zero. When the frequency is zero, the modal analysis of the frame is not meaningful. And from the table we can find the natural frequency of each frame frame. Next, the 4-10 order mode of the frame is analyzed.

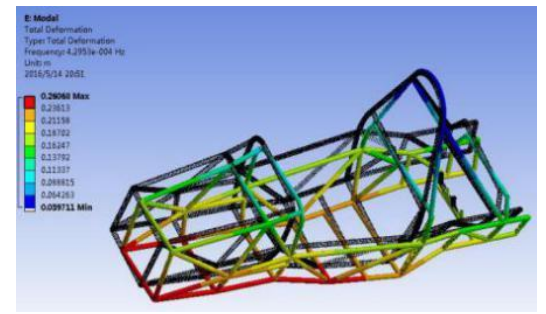

Fig.4 4 order displacement

It can be seen from Figure 4 that the vibration mode of the frame is approximately shifted to the left and the maximum displacement is the front left lower truss. The vibration frequency of the frame is low, which corresponds to the rigid body translation of the frame vibration.

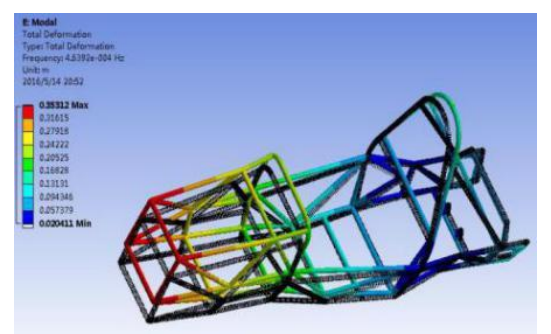

Fig. 55 order displacement 
It can be seen from Fig. 5 that the 5th order vibration mode of the frame is the deformation of the front head and rear rear, similar to the state of braking, and the maximum displacement is at the front of the frame. Frame natural frequency is low.

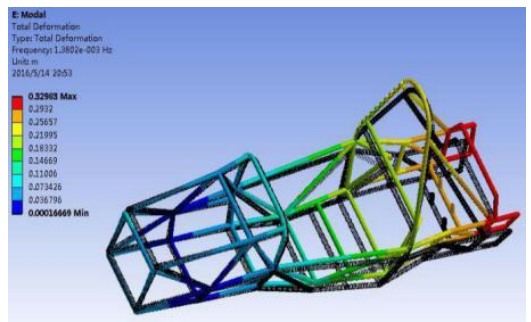

Fig. $6 \quad 6$ order displacement

It can be seen from Figure 6 that the vibration of the frame is nodal motion deformation. The maximum displacement is at the tail of the frame.

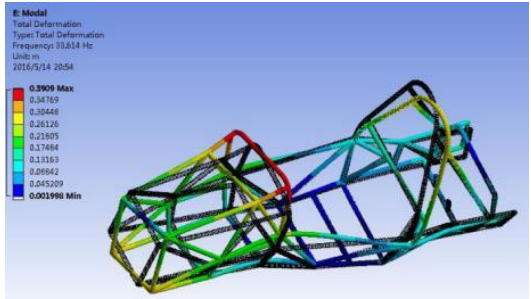

Fig. 77 order displacement

It can be seen from Figure 7 shows the 7-order vibration mode for the front frame to the right front overhang direction of bending deformation, the rear right rear overhang bending deformation, the overall $\mathrm{S}$ deformation. The natural frequency of the frame is $33.614 \mathrm{~Hz}$.

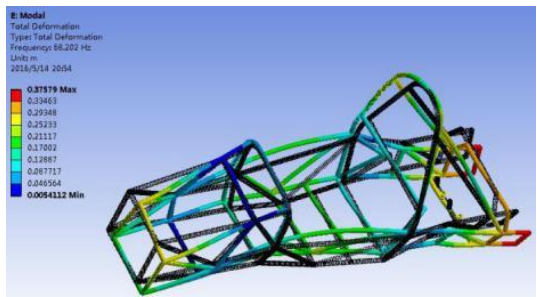

Fig. 88 order displacement

It can be seen from Figure 8, 8-stage vibration mode is similar to do V-shaped swing deformation.The natural frequency of the frame is $68.202 \mathrm{~Hz}$.

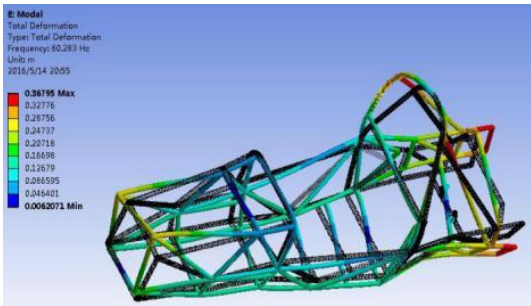

Fig. 99 order displacement

It can be seen from Figure 9, 9 frame mode is similar to the deformation of the torsional movement. The natural frequency of the frame is $80.283 \mathrm{~Hz}$.

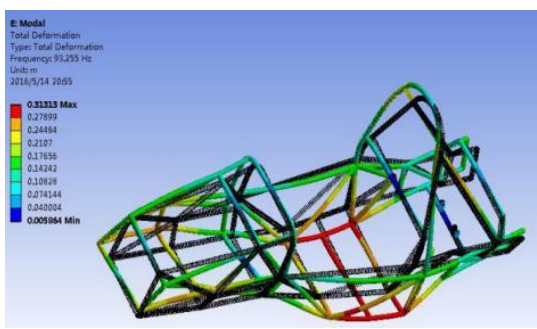

Fig. 1010 order displacement

It can be seen from Fig. 10 that the vibration mode of the frame 10 exhibits a front-rear distortion. The natural frequency of the frame is $93.255 \mathrm{~Hz}$.

\section{Conclusions}

According to the vibration mechanics, the natural frequency and the mode shape depend on the physical parameters such as M-matrix and $\mathrm{K}$-matrix of the system, where $\mathrm{M}$ is the mass matrix and $\mathrm{K}$ is the stiffness matrix. The structure of M-matrix and K-array is affected by factors such as the arrangement of the parts in the structure, the quality and the cross-sectional shape. Therefore, by changing the geometry of the structure, the form of the M-matrix and the K-matrix can be changed, thereby changing the natural frequency of the structure and finally avoiding the resonance.

The frequency of the engine can be calculated by the following formula:

$$
f=\frac{2 n z}{60 r}
$$

In the formula:

$\mathrm{n}$--the engine speed; 
z--the number of engine cylinders;

r--the number of engine strokes.

The 4-cylinder 4-stroke engine is selected by most FSCs. The engine idling speed is $1000 \mathrm{r} / \mathrm{min}$, you can calculate the idle frequency is $33.3 \mathrm{~Hz}$. The normal speed is $5000-7000 \mathrm{r} / \mathrm{min}$, then the frequency range is $166.7-233.3 \mathrm{~Hz}$. From the results of modal analysis can be drawn frame 1-10 of the natural frequency are below $100 \mathrm{~Hz}$, well to avoid the excitation frequency of the engine, so the frame will not reach resonance.

\section{References}

[1] Ji Fei,Li Jing,Ma Zehong.Freedom Modal Analysis for FSAE Car Frame

[J].Tractor\&FarmTransporter.2014,41(6):26-27.

[2] Wang Minglei,Dai Zuqiang,Sheng Jian.Modal Anlysison Natural Gas Bus Frame Structure Based on Ansys

Workbench[J].Bus\&Coach Technology and Research.2015(1):9-11.

[3] Wang Bing,Liu Yunhuan.Modal Analysis of FSAE Frame and Lightweight Design by ANSYS

Workbench[A].2013CN-ET093:613-614.

[4]Ma Xun,Sheng Yongsheng.Finite element analysis and optimization of frame stiffness and modal[J].Bus Technology and Research.2004,26(4):9-11.

[5] Sachin Sudhakar Harak,Satish Chand Sharma,Suraj Prakash Harsha. Modal analysis of prestressed draft pad of freight wagons using finite element method[J]. Journal of Modern Transportation.2015(01). 